\title{
The Relationship between Body Image Satisfaction and Bulimia Nervosa among King Saud University Students
}

\author{
Suliman Saleh Aljomaa ${ }^{1}$ \\ ${ }^{1}$ Department of Psychology, College of Education, King Saud University, Riyadh, Saudi Arabia \\ Correspondence: Suliman Saleh Aljomaa, Department of Psychology, College of Education, King Saud \\ University, Riyadh, Saudi Arabia. E-mail: jomaa@ksu.edu.sa
}

Received: January 11, 2018

Accepted: March 1, $2018 \quad$ Online Published: April 27, 2018

doi:10.5539/ies.v11n5p123

URL: https://doi.org/10.5539/ies.v11n5p123

\begin{abstract}
The study aimed at examining the relationship between body image satisfaction and bulimia nervosa among the students of education faculty at king said university students. The author used the tests of bulimia nervosa and body image test. The researcher verified tests reliability. Students from King Saud University randomly selected (No. 337) participated in the study. Overall mean of the body image satisfaction test scored 3.66 degrees, and the overall mean of the bulimia nervosa test scored 3.42 degrees. Data analysis indicated that there is a positive correlation between the body image satisfaction and bulimia nervosa, Pearson correlation coefficient scored 0.648 , and bulimia nervosa predicted body image.
\end{abstract}

Keywords: bulimia nervosa, anorexia nervosa, body image satisfaction, eating disorder

\section{Introduction}

The way we look to our body occupied our minds, and reflected clearly on our psychological state, self-satisfaction, and sociopsychological adjustment. The individual is either satisfied about his body image and this helps in adjusting or not satisfied and it leads to maladjustment. Body image represents the mental image, which an individual composes about his body and external appearance. Al-Kafafi and Al-Nayal (1995) found a correlation between the body image and the individuals' psychological state and concern about appearance in front of the others. In the same respect, Jaber and Al-Kafafi (1999) defined the body image as the overall physical and functional features individuals self-create (body perception) and their attitudes toward these features (body concept), this image emerges from conscious and unconscious sources and constitute a basic component in our self-perception. However, Shqair (1999) believes the body image is a mental image developed by the individual about his body, whether it is the external appearance or the body internal components, and his ability to recruit these organs and prove their efficacy with the positive or negative feelings accompanying the mental image about the body. Therefore, scholars define body image as the individuals' perception about his body and the way he appears in social and cultural contexts. It refers to the external appearance of the body in terms of individuals' evaluation of his body appearance, tidiness and perception of size and weight (Knott, Woodward, Hoefkens, \& Limbert, 2015)

Body image has two concepts: the first is the body ideal; it means the attractive and proper body style for a certain age group, accepted in the individual culture. The other concept is body concept; it includes ideas, beliefs, and limitations correlated with the body, in addition to the body perception (Jaber \& Al-Kafafi, 1991)。

Body image satisfaction occupies female's minds more than males, for that reason it is clearer among females, while males are more interested in their professional future and achievement. Satisfaction is influenced by others evaluations, a women for instance considers her husband satisfaction about her body, while her own body image satisfaction is achieved by being attractive in her husband eyes, and by maintaining the proper weight from her own point of view based on the prevalent social norms. The proper weight is the cornerstone in body image components among females in different cultures, followed by face image and body image satisfaction pursued by males and females in different levels. While males focus on upper body image and muscles, females take care of their body as a whole (Zuhair, 2007).

Culture contribute to a large extent in the individuals' perceptions of his body, more attuned image to the cultural standards in terms of body attractiveness leads to more body self-image satisfaction. Culture emphasis on certain 
body dimensions vary. Certain cultures emphasize the length of the body and parts size among males and females, these features are signs of strength and dignity. Other cultures emphasize the physical health. However, most cultures favor weight and size over the standard among males compared to less than the standard among females. In this case, males' body image satisfaction scores more if the body weight and size is over the standard while females body image satisfaction scores more if their weight and size is below the standard.

Psychologically body image among female adolescents correlates with anorexia nervosa; its symptoms include poor appetite, severe weight loss, and weakness. Scientists believe that body image among them is characterized by distortion and disorder. On the other hand, bulimia nervosa correlated largely with the body image (Masha'el, 2010). Females consume lots of food and drinks; depression follows, anxiety, and hypochondriacally neurosis because their obesity leads to body image distortion. (Koff, Stabbs, \& Redam, 1990; Poulsen et al., 2014)

Many theories explained anorexia nervosa, The biological theory advocates found that the first kens of anorexia nervosa patients (degree 1) are more likely to be have bulimia nervosa, Anorexia nervosa patients affect closed relatives up to eight times compared with individuals other than relatives (Shqair, 1999). The prevalence of the disorder of an infected family member with his brothers and sisters or twins percentage scored (6-10\%) compared to (1-2\%) with nonrelatives (Dosoqi, 2006; Othman, 2001).

Anorexia nervosa organic reasons include:

1) Chemistry disorders, such as hormonal disorders (genetic or caused by thyroid gland enlargement) that affects the metabolism.

2) Brain cell damage, especially the cells responsible for hunger and fullness feelings (hypothalamus), when this region is damaged the patient does not feel full and consumes more food leading to an increase in his weight. Brain tumor patients and brain damage patients in the hypothalamus region increase their weight.

3) Taking Antidepressant and antihistamine medications may increase appetite and leads to hyperthyroidism.

Few studies supported the assumption that bulimia nervosa patients show deficiency in the serotonin activity. While other studies found no significant differences in the serotonin activity between bulimia nervosa patients and the control groups (Othman, 2001). Anemia, diabetes, pregnancy convulsions, placenta infarction, birth heart problems, and birth problems correlates with bulimia nervosa.

A study published in 198) in the British medical journal (cited by Ahmad, 2013) indicated that (6-10\%) of the bulimia nervosa female sisters patients that have the same genetic factors increased between identical twins. Hormonal disorders were responsible for the hypothalamus disorders that precede the weight loss in $20 \%$ of the patients.

Frustration releases occur according to the psychoanalysis theory by eating lots of food. The theory attributes excessive eating to the individuals' exposure to internal and external conflict, so bulimia becomes the way to express aggression because of depressed feeling. (Al-Omar \& Al-Dghaim, 2007)

The striking study of Koff, Rierdan, and Stubbs (1991) explored the correlation between body image and the self-concept among American $9^{\text {th }}$ grad (male and female) students (No. 169). The researchers utilized the self-concept and body image tests. Data analysis illustrated that females body image concept is different from males, males were more positive in their self-perceived body image compared to females as was assumed. Contrary to expectations, the correlation between body image and self-concept differences attributed to gender were not functional, nevertheless the correlation scores were higher among males compared to females.

Straeter (2003) studied the correlation between body image and bulimia nervosa. Participants (57 female undergraduate) allocated to two groups based on the body image test: positive body image and negative body image completed the body image test. The participants of the negative body image had some properties that made them venerable to eating disorders.

Trautmann, Worthy, and Lokken (2007) confirmed that the individuals' satisfaction of his overall body degree is a key factor and an indicator in forming the body image, self-concept and self-confidence. People often compare their body with the body ideal found in the society. Females are less satisfied about their bodies and more interested in their figures compared with males. They also studied the correlation between body image and bulimia nervosa. The result was, unsatisfied body image females compared to males, they are also more self-criticizers.

Zuhair (2007) studied the sources of adolescents' body image and its correlation with some demographical variables. The participants were selected from the seventh, eighth and Eleventh grades total number of the participants (208) students. The variables of gender, ideal weight parents, and family attitudes correlated and influenced the body image degree; females' body image scores were higher than males' scores. Younger male 
students were less satisfied about their body image while the same category of female students were more positive and body image satisfied, partners and media had a negative impact on the students body image. The researcher found a positive correlation between the academic achievement and the body image evaluation as a whole, and in favor of the higher achievers. The size of the body was the most correlated followed by media and length of the body. Finally, peers influenced female students more compared with males.

Al-Shoqran (2009) investigated the correlation between body image and eating disorders among a selected group of adolescents (No. 408) from Ramtha- Jordan. The satisfaction degree scored averaged and the level of eating disorders was low. The researcher found functional correlations between the level of eating disorders and body image satisfaction.

Masha'el (2010) investigated females' body image correlation with depression, social anxiety, and self-appreciation. Females participating in the study (No. 638) showed a correlation between perceived body image satisfaction degree and self-appreciation.

In a similar respect, Melhem (2012) investigated the effect of eating disorders, social anxiety, and obsessive-compulsive disorder correlation with body image satisfaction among a group of adolescents from Jordan. Social anxiety, eating disorders, and obsessive-compulsive disorder influenced the level of body image satisfaction. The researcher found that body image significant differences attributed to gender.

As-Sa'edi, Sheerah, Al-Ayoubi, Tajaddin, and Habeeb (2013) investigated body image satisfaction among medicine students at Taibah University in Madinah, KSA. The students (No. 242) were not satisfied about their body image, the dissatisfaction correlated with the body mass index, the students desired to be thinner. Family, friends, social view, and feelings body parts imbalance affected body image satisfaction.

Smink et al. (2016) investigated bulimia nervosa and its correlation with anorexia nervosa. The purpose was to study changes in eating disorder occurrence in Netherlands during 1985-1989, 1995-1999, and 2005-2009. The rates of overall bulimia nervosa patients decreased in the last three decades, (from 8.6 for every 100000 in 1985-1989 to 6.1 in 1995-1999 to 3.2 in 2005-2009).

In the same respect, Molbert et al. (2017) investigated the body image correlation with bulimia nervosa. Participants (926) body image correlated with bulimia nervosa, which in turn predicted body image.

Franko et al. (2018) conducted a longitudinal study to investigate predictions of eating disorder for 22 years. Two hundred twenty eight females participated in the study from those one hundred and sixty seven stayed a life of which 100 were diagnosed with anorexia nervosa and the rest were diagnosed with bulimia nervosa, after 20-25 years and were interviewed for a second time to assess the change and patients recovery. The researcher diagnosed the students with deep depression after 22 years.

The previous studies investigated body image or bulimia nervosa with different populations, sample size, and results. The variables studies varied such as social anxiety, compulsive disorder, and self-concept. Body image and compulsive disorder correlation was investigated by Molbert at al. (2017); Trautmann, Worthy, and Lokken (2007) and Straeter (2003) in foreign societies. Scarcity of studies on Arab environment motivated the author to conduct the current study.

\subsection{Study Problem}

The bulimia nervosa is the relationship between the individuals' psychological state and a whelming desire to eat or not to eat; this relationship is not new, and known for a long time. The desire to eat is a way to express nervousness and disorder. Scientists termed bulimia nervosa a psychometric disorder before the DSM-IIIR emergence in (1987), then DSM-IV emerged in (1994) followed by Diagnostic and Statistical Manual of Mental Disorders (DSM-IV-TR) and gave bulimia nervosa a separate category including bulimia nervosa, anorexia nervosa, and other unclassified eating disorders.

Body image is an important psychological manifestation that negatively affects individuals' life, the exposure to unusual circumstances or illnesses such as bulimia nervosa causes a disorder of body image. Body image is the mental image an individual forms about his or her body, whether it is the external appearance or the body internal components, and his ability to recruit these organs and prove their efficacy with the positive or negative feelings accompanying the mental image about the body.

Food eating influences human behavior, muscular and mental activity, it also influence the psychological and physical state. The modern society became obsessed with the body mass, fears obesity and its health complications, because of medical and food awareness, and because heart diseases spread, common cycosomatic diseases and cardiac complications (Al-Shoqran, 2009). 
The study attempted to investigate the correlation between body image satisfaction and bulimia nervosa by answering the following questions:

1) What is the level of body self-image satisfaction among the students of the education faculty at King Saud University?

2) What is the level of bulimia nervosa among the students of the education faculty at King Saud University?

3) Is there a functional correlation between body image satisfaction and bulimia nervosa?

4) Is body image predictable by bulimia nervosa?

\subsection{Study Importance}

The study investigated an important topic in shaping a healthy identity, and helping in sociopsychological adjustment with the surrounding. It examined the correlation between body image and bulimia nervosa and recommended of healthy diets. Investigating the correlation between body image and bulimia nervosa may help individuals interested in this domain to lay sound foundations to prevent obesity and correlated health and psychological diseases.

\subsection{Study Goals}

The study aims at:

1) Identifying the level of body self-image satisfaction among the students of the education faculty at King Saud University.

2) Identifying the level of bulimia nervosa among the students of the education faculty at King Saud University.

3) Investigating the correlation between body image satisfaction and bulimia nervosa.

4) Investigating the predictability between body image satisfaction and bulimia nervosa.

\subsection{Procedural Definitions}

Body Image is defined as the overall physical and functional features individuals self-create (body perception) and their attitudes toward these features (body concept), this image emerges from conscious and unconscious sources and compose a basic component in our self-perception (Melhem, 2012) procedurally it is the degree obtained on the body image test.

Eating Disorders, is the extreme attitudes and behaviors concerned with eating and weight, it comes in two types: bulimia nervosa and anorexia nervosa and it reflects psychological and physiological problems that may have dangerous and damaging effects on its patients (Al-Zaghaleel, 2009). Procedurally it is the degree obtained on eating disorder test.

\subsection{Study Limitations}

Generalizing the outcomes of the current study is inhibited by the tests (eating disorder and body image satisfaction) psychometric properties and implementation duration. Therefore, results are valid to generalize on similar contexts.

\section{Methodology}

A descriptive correlative approach is used in the study.

\subsection{Study Sample}

Male and female students from the faculty of education at King Saud University enrolled in the academic year 2015-2016 participated in the study. Participants overall number was 337 students.

\subsection{Instruments of the Study}

The study used the tests of body image satisfaction and eating disorders.

\subsubsection{Bulimia Nervosa Test}

The researcher adopted the bulimia nervosa test designed by Shqair (1999). The test included 24 items, the student selects based on his certainty degree the answer from the statements in front of each item (Yes, more or less and no).

Content Validity: Nine professors at King Saud University majoring in educational psychology reviewed the final version of the test. They provided remarks to the test in terms of structure, clarity, suitability, and appropriateness to measure its dimension, relevancy to an Arab context and age group. Based on the remarks provide few items structures were modified, the reviewers agreed by $80 \%$ on the test validity for the purpose of measuring bulimia 
nervosa among the selected sample.

Construct Validity: The test was implemented on a pilot sample of (26) students. Correlation coefficients of each item were computed with the overall test the scores range between (0.39-0.87) as illustrated in Table 1.

Table 1. Correlation coefficients between the items and the overall degree

\begin{tabular}{cccccc}
\hline Item No. & Correlation coefficient & Item No. & Correlation coefficient & Item No. & Correlation coefficient \\
\hline 1 & $.66^{* *}$ & 9 & $.39^{* *}$ & 17 & $.39^{* *}$ \\
2 & $.65^{* *}$ & 10 & $.60^{* *}$ & 18 & $.67^{* *}$ \\
3 & $.63^{* *}$ & 11 & $.62^{* *}$ & 19 & $.62^{* *}$ \\
4 & $.72^{* *}$ & 12 & $.49^{* *}$ & 20 & $.49^{* *}$ \\
5 & $.59^{* *}$ & 13 & $.61^{* *}$ & 21 & $.65^{* *}$ \\
6 & $.55^{* *}$ & 14 & $.61^{* *}$ & 22 & $.62^{* *}$ \\
7 & $.85^{* *}$ & 15 & $.81^{* *}$ & 23 & $.80^{* *}$ \\
8 & $.58^{* *}$ & 16 & $.85^{* *}$ & 24 & $.39^{* *}$ \\
\hline
\end{tabular}

Table 1 illustrated that all the correlation coefficient scores were acceptable and functional; therefore, the researcher excluded none of the items.

Test Reliability: To establish the reliability of bulimia nervosa test it was implemented on a pilot sample of 26 students, data collected were analyzed using the Cronbach Alpha equation, the result obtained was $(0.85)$ was considered appropriate for the study. A pilot sample completed the test twice with an interval of two weeks to verify the bulimia nervosa test retest reliability. The estimates of the two tests obtained were computed by implementing Pearson correlation and it scored (0.87) degrees.

\subsubsection{Test Correction Procedure}

The test included 24 items, students were instructed to select from 1-3 on a 3-point Likert scale (yes, more or less, no) the answer based on the degree of their certainty about the item. The yes answer took three points; more or less got two points and no took one point.

Body Image Test. The researcher adopted the test Arabized by Dosoqi (2006) in the current study, it included 18 items, students select based on their certainty about the item one of six alternatives.

Body Image Test Validity: Body Image Test Content Validity. Nine professors at King Saud University majoring in educational psychology insured the test validity. They provided remarks to the test items in terms of structure, clarity, suitability, and appropriateness to measure its dimension, relevancy to an Arab context and suitability to the age group. Based on the remarks provide few items structures was modified, the reviewers agreed by $82 \%$ on the test validity for the purpose of measuring bulimia nervosa among the selected sample.

Body Image Test Construct Validity: The test was implemented on a pilot sample of 26 students. Correlation coefficients of each item were computed with the overall test the scores range between 0.43 and 0.81 as illustrated in Table 2.

Table 2. Correlation coefficients between the items and the overall degree of the body image test

\begin{tabular}{cccc}
\hline Item No. & Correlation coefficient & Item No. & Correlation coefficient \\
\hline 1 & $.62^{* *}$ & 10 & $.43^{* *}$ \\
2 & $.55^{* *}$ & 11 & $.63^{* *}$ \\
3 & $.63^{* *}$ & 12 & $.62^{* *}$ \\
4 & $.72^{* *}$ & 13 & $.47^{* *}$ \\
5 & $.54^{* *}$ & 14 & $.61^{* *}$ \\
6 & $.57^{* *}$ & 15 & $.61^{* *}$ \\
7 & $.85^{* *}$ & 16 & $.81^{* *}$ \\
8 & $.58^{* *}$ & 17 & $.80^{* *}$ \\
9 & $.80^{* *}$ & 18 & $.49^{* *}$ \\
\hline
\end{tabular}

Table 2 illustrated that all the correlation coefficient scores were acceptable and functional; therefore, the researcher excluded none of the items. 
Test Reliability: to establish the reliability of body image test it was implemented on a pilot sample of 26 students, data collected were analyzed using the Cronbach Alpha equation, the result obtained was $(0.81)$ was considered appropriate for the study. The researcher subjected the pilot sample to the bulimia nervosa test twice with an interval of two weeks to verify its reliability. The estimates of the two tests obtained were computed by implementing Pearson correlation and it scored (0.86) degrees.

Test Correction Procedure: The test included 18 items, students were instructed to select from answers on a 5-point Likert scale based on their agreement from: (1) Strongly disagree; (2) Disagree; (3) Neither agree nor disagree; (4) Agree; (5) Strongly agree.

The points were categorized as follows: very low (1- less than 1.80); low (1.81-2.60); average (2.61-3.40); high (3.41-4.20) and very high (4.21-5)

\subsection{Study Procedure}

Validity and reliability of bulimia nervosa test and body image test were established. The researcher obtained the total number of the students enrolled in King Saud University for the academic year 2015-2016 from the lists of students at the registration deanship. The total number represented the population of the study; the sample selection was in random. The students received the tests papers tests and instructions to select items answers. Data collection and analyses lasted for three weeks.

\subsection{Study Variables}

The study included the variables of body image satisfaction and bulimia nervosa and gender.

\subsection{Data Analysis}

Data was analyzed by computing means, standard deviations, Pearson and regression analyses of the two tests.

\section{Results and Discussion}

The study attempted to investigate the correlation between body image satisfaction and bulimia nervosa by answering its questions.

To answer the first question of "What is the level of body self-image satisfaction among the students of the education faculty at King Saud University?" the researcher computed means and standard deviations of King Saud University student's body image satisfaction as Table 3 illustrates.

Table 3. Means and standard deviations of the students body image satisfaction

\begin{tabular}{ccc}
\hline Items & M. & SD. \\
\hline 1 & 3.45 & 1.172 \\
2 & 3.95 & .706 \\
3 & 3.80 & 1.206 \\
4 & 2.29 & 1.191 \\
5 & 3.12 & 1.298 \\
6 & 2.73 & 1.355 \\
7 & 3.03 & 1.380 \\
8 & 3.18 & 1.002 \\
9 & 3.47 & 1.190 \\
10 & 2.09 & 1.137 \\
11 & 2.59 & 1.225 \\
12 & 2.18 & 1.156 \\
13 & 2.44 & 1.230 \\
14 & 2.24 & 1.215 \\
15 & 3.38 & 1.125 \\
16 & 2.15 & 1.227 \\
17 & 1.65 & .970 \\
18 & 3.30 & 1.264 \\
Overall & 3.66 & .521 \\
\hline
\end{tabular}

As observed in Table 3 means of the students responses scores on the body image satisfaction was 1.65-3.95, the overall mean score was 3.66 , this result refers to a positive body image.

Appearance of the individual is an important factor in relationships and life in general, the body image is important 
in psychological and physical development, it has a symbolic importance as well. Anxiety correlated with the body image; it plays a vital role in professional decision-making, self-efficacy, and determination. Negative body image correlates with anxiety, disgust, despair, anger, and shyness.

The appearance of the individual importance supposes an important correlation between the psychological state and body image evaluations, positive recognition of the body image helps to think the self is attractive, which is important for personality maturity and development. Positive body image believers have positive body images.

To answer the second question of "What is the level of bulimia nervosa among the students of the education faculty at King Saud University?" means and standard deviations were computed as Table 4 illustrated.

Table 4. means and standard deviations of the bulimia nervosa test

\begin{tabular}{ccc}
\hline Items & M. & SD. \\
\hline 1 & 2.48 & 1.190 \\
2 & 3.28 & 1.535 \\
3 & 3.13 & 1.389 \\
4 & 2.40 & 1.174 \\
5 & 2.69 & 1.337 \\
6 & 2.43 & 1.153 \\
7 & 2.99 & 1.274 \\
8 & 2.65 & 1.287 \\
9 & 2.83 & 1.223 \\
10 & 2.55 & 1.284 \\
11 & 2.21 & 1.101 \\
12 & 2.64 & 1.201 \\
13 & 1.68 & 1.020 \\
14 & 2.79 & 1.428 \\
15 & 2.25 & 1.148 \\
16 & 2.70 & 1.188 \\
17 & 2.67 & 1.271 \\
18 & 1.64 & .958 \\
19 & 1.54 & .918 \\
20 & 1.84 & 1.127 \\
21 & 1.53 & .895 \\
22 & 2.79 & 1.378 \\
23 & 2.42 & 1.303 \\
24 & 2.96 & 1.494 \\
Overall & 3.42 & .838 \\
\hline & &
\end{tabular}

As observed in Table 4 means of the students responses scores on the bulimia nervosa test was $1.53-3.28$, the overall mean score was 3.42 .

Adolescents and youth eating disorders percentage scores up to $8.22 \%$. Eating disorders can be characterized by starving and self-controlled eating until the state of appetite loss is reaches or by eating too much. Eating disorders spread in most societies nowadays as a result many diseases in different forms such as psychological, physical, or psychophysical diseases emerged. The high percentage of eating disorders among the participants of the study may be attributed to the fear of severe obesity and strive to be thin. (Al-Abadsah, 2013)

To answer the third question of "Is there a functional correlation between body image satisfaction and bulimia nervosa?" the researcher used Pearson correlation coefficient to compute the correlation between body image satisfaction and bulimia nervosa. The correlation was functional at 0.000 and it scored 0.648 .

Both the disorders of bulimia nervosa and anorexia nervosa lead to physical and psychological effects; have serious consequences on the individual physical and psychological health such as increased anxiety, withdrawal, feelings of social isolation and depression. Body image dissatisfaction decreases self-esteem, leads to weakness in the ability to control motivation and needs, tension, emotional imbalance, obsessive-compulsive disorders, concerns about over weight and family relations decrease (Al-Kafaee, 2013). It is observed that both bulimia nervosa and body image new topics that have emerged by social and cultural developments. The prevailing 
cultural values play a prominent role in the society attention toward body image; societies differ in their standards about body image (Al-Nabulsi, 2003).

Previous research proved that females exaggerate in their body image evaluations, they see themselves more fat than they actually are and try to reduce their weight to reach the ideal image, to be able to feel self-achievement especially when thin is considered the current time culture and one of women beauty standards. (Ahmad, 2013)

Modern societies concern about food issues is increasing and at the same time the obesity ratios are increasing and the issue is attracting individuals, families, institutions, physicians, psychologists and sociologists attention. In ancient times, obesity was a beauty sign, good health, happiness, and economic wellbeing sign, but it turned to be an indicator of lack of health education among individuals, families, or communities (Izgic, Akyus, Dogan, \& Kugu, 2004).

The opposite of health concern, more restaurants are opening every day, TV cooking shows are attracting more people to buy and try, especially among adolescents, and in the same time gaining weight among adolescents body builders is also spreading, they start eating too much until they couldn't stop, this leads to overweight. Insurance companies started thinking of solutions for the problem because overweight and obesity leads to sudden deaths, this will make these companies suffer from heavy loss. Mal eating behavior motivated those who were concerned with good health to care for diet programs.

Clinical descriptions of bulimia nervosa go back to hundreds of years. It appeared for the first time in the Diagnostic and Statistical Manual of Mental Disorders, Fifth Edition published by the American psychiatric association in 1980 as a disorder type that start in childhood and adolescence. In the fourth edition published, bulimia nervosa took an independent category from psychological disorders (Rodin, 1993).

To answer the final question of the study "Is body image predictable by bulimia nervosa?" the results obtained were computed by using linear regression the result was $(\mathrm{R} 2=0.42), \mathbf{F}$ value was functional at $(0.000)$ and it scored (213.95). The result confirmed that bulimia nervosa has an effect on the body image; the following predictable formula illustrates this.

\section{Body image $=-5.84+1.27 *$ bulimia nervosa}

Obesity among female adolescents is a source of fear. Adolescent's attempts to lose weight associated with depressive symptoms such as anxiety and obsession of recognizing the overweight effect or the self-image that influence self-evaluation or thought evaluation because of the seriousness of body weight.

Scholars have noticed two groups of anorexia nervosa patients, the first group loses weight by reducing eating they compose $60 \%$ of the anorexia nervosa patients, the second group eats too much then purges to prevent the body from absorbing food by purging, taking laxatives and diuretics. The main characteristic that distinguishes anorexia nervosa is fear of weight gain that does not appear in other disorders (Dakhil, 2007).

\section{Recommendations}

Based on the results of this study the researcher calls for designing treatment programs to improve the body image of those who are not satisfied with their body image. Scholars should conduct further studies on different samples and age groups; therefore, it is necessary to qualify psychological advisors to be able to help eating disorder patients.

\section{References}

Ahmad, N. F. (2013). Body image as a determinant of some psychological construction dimensions among children with obesity. The journal psychological studies in Arabic, 12(1), 182-222.

Al-Abadsah, A. A. A. (2013). Body image satisfaction and its relationship with depression among Palestinian female adolescents in Gaza strip. Islamic University Journal for Educational and Psychological Studies, $12(3), 867-888$

Al-Kafaee, H. (2013). Body and its relationship with social acceptance (Unpublished master's thesis). Qadisiya University, Iraq.

Al-Kafafi, A., \& Al-Nayal, M. (1995). Body image and some personality variables among a group of female adolescents: a developmental correlative cross-cultural study. Alexandria: Dar_almarefa.

Al-Nabulsi, M. A. (2003). Obesity the causes and treatment. The journal of psychological education, 7(5), 33-45.

Al-Omar, B. O., \& Al-Dghaim, M. D. (2007). The structural model of emotional sign of the psychological stress. Educational Journal, 82(5), 144-167. 
Al-Shoqran, H. (2009). Body image and its relationship with eating disorders and self-esteem among a sample of female adolescents in Ramtha (Unpublished master's thesis). Yarmouk University. Jordan.

Al-Zaghaleel, A. S. (2009). Eating disorders among the university students and the differences in it according to some demographical variables. Journal of Educational and Psychological Sciences, 10(1), 90-123.

As-Sa'edi E., Sheerah, S., Al-Ayoubi, R., Al-Jehani, A., Tajaddin, W., \& Habeeb, H. (2013). Body image dissatisfaction: prevalence and relation to body mass index among female medical students in Taibah University, J. Taibah Univ Med Sci., 3(8), 126-33.

As-Sa'edi, E., Sheerah, S., Al-Ayoubi, R., Tajaddin, W., \& Habeeb, H. (2013). Body image dissatisfaction: prevalence and relation to body mass index among female medical students in Taibah University, 2011. Journal of Taibah University Medical Sciences, 8(2), 126-133. https://doi.org/10.1016/j.jtumed.2013.05.001

Dakhil, M. S. (2007). Body image and its relation with anorexia nervosa and bulimia nervosa among female students at King Saud University (Unpublished master's thesis). King Saud University. KSA

Dosoqi, M. M. (2006). Body image disorders: Causes, diagnosis, prevention and treatment. Cairo: Anglo-Egyptian Library.

Franko, D. L., Tabri, N., Keshaviah, A., Murray, H. B., Herzog, D. B., Thomas, J. J., ... \& Eddy, K. T. (2018). Predictors of long-term recovery in anorexia nervosa and bulimia nervosa: Data from a 22-year longitudinal study. Journal of Psychiatric Research, 96, 183-188. https://doi.org/10.1016/j.jpsychires.2017.10.008

Izgiç, F., Akyüz, G., Doğan, O., \& Kuğu, N. (2004). Social phobia among university students and its relation to self-esteem and body image. The Canadian Journal of Psychiatry, 49(9), 630-634. https://doi.org/10.1177/070674370404900910

Jaber, J. A. H., \& Al-Kafafi, A. (1999). Dictionary of Psychology and Psychiatry (Vol. 2). Arab Renaissance, Egypt.

Knott, S., Woodward, D., Hoefkens, A., \& Limbert, C. (2015). Cognitive behaviour therapy for bulimia nervosa and eating disorders not otherwise specified: translation from randomized controlled trial to a clinical setting. Behavioural and cognitive psychotherapy, 43(6), 641-654. https://doi.org/10.1017/S1352465814000393

Koff, E., Rierdan, J., \& Stubbs, M. L. (1990). Gender, body image, and self-concept in early adolescence. The Journal of Early Adolescence, 10(1), 56-68. https://doi.org/10.1177/0272431690101004

Masha'el, T. (2010). Women body image and it relationship with depression, social anxiety and self-esteem: A diagnostic study on a sample in Latakia (Unpublished Doctoral thesis). Latakia University. Syria.

Melhem, S. M. (2012). The effect of eating disorders, social anxiety, obsessive compulsive disorder and self-esteem in body image satisfaction among o group of adolescents in Jordan. Journal of Banha Faculty of Education, 90, 112-138.

Mölbert, S. C., Klein, L., Thaler, A., Mohler, B. J., Brozzo, C., Martus, P., ... \& Giel, K. E. (2017). Depictive and metric body size estimation in anorexia nervosa and bulimia nervosa: A systematic review and meta-analysis. Clinical Psychology Review, 57, 21-31. https://doi.org/10.1016/j.cpr.2017.08.005

Othman, F. S. (2001). Anxiety and psychological stress management. Arab Thought House, Cairo.

Poulsen, S., Lunn, S., Daniel, S. I., Folke, S., Mathiesen, B. B., Katznelson, H., \& Fairburn, C. G. (2014). A randomized controlled trial of psychoanalytic psychotherapy or cognitive-behavioral therapy for bulimia nervosa. American Journal of Psychiatry, 171(1), 109-116. https://doi.org/10.1176/appi.ajp.2013.12121511

Rodin, J. (1993). Cultural and psychosocial determinants of weight concerns. Annals of internal medicine, 119(7_Part_2), 643-645. https://doi.org/10.7326/0003-4819-119-7_Part_2-199310011-00003

Shqair, Z. (1999). The effectiveness of cognitive behavioral therapy in treating bulimia nervosa among the university female students. Psychological Studies, 9(4), 515-557.

Smink, F. R., van Hoeken, D., Donker, G. A., Susser, E. S., Oldehinkel, A. J., \& Hoek, H. W. (2016). Three decades of eating disorders in Dutch primary care: decreasing incidence of bulimia nervosa but not of anorexia nervosa. Psychological medicine, 46(6), 1189-1196. https://doi.org/10.1017/S003329171500272X

Straeter, S. V. (2003). Body image and acculturation status, eating disorder symptomatology, psychopathology and self-esteem in Latina college students.

Trautmann, J., Worthy, S. L., \& Lokken, K. L. (2007). Body dissatisfaction, bulimic symptoms, and clothing 
practices among college women. The Journal of Psychology, 141(5), 485-498. https://doi.org/10.3200/JRLP.141.5.485-498

Zuhair, A. H. (2007). Body image among adolescents its sources and relation with demographical variables (Unpublished Doctoral thesis). Jordan University. Jordan.

\section{Note}

Note 1. This is a research project that was supported by a grant from the research center for college of education, deanship of scientific research at King Saud University.

\section{Copyrights}

Copyright for this article is retained by the author(s), with first publication rights granted to the journal.

This is an open-access article distributed under the terms and conditions of the Creative Commons Attribution license (http://creativecommons.org/licenses/by/4.0/). 